\title{
Modelling Blast Effects on a Reinforced Concrete Bridge
}

\author{
Markellos Andreou, ${ }^{1}$ Anastasios Kotsoglou, ${ }^{1}$ and Stavroula Pantazopoulou ${ }^{2}$ \\ ${ }^{1}$ Department of Civil Engineering, Laboratory of Reinforced Concrete, Democritus University of Thrace, \\ V. Sofias 12, 67100 Xanthi, Greece \\ ${ }^{2}$ Department of Civil Engineering, The Lassonde Faculty of Engineering, York University, 4700 Keele Street, \\ Toronto, ON, Canada M3J 1 P3 \\ Correspondence should be addressed to Stavroula Pantazopoulou; stavroula.pantazopoulou@gmail.com
}

Received 21 April 2016; Revised 27 June 2016; Accepted 28 June 2016

Academic Editor: Chiara Bedon

Copyright ( $) 2016$ Markellos Andreou et al. This is an open access article distributed under the Creative Commons Attribution License, which permits unrestricted use, distribution, and reproduction in any medium, provided the original work is properly cited.

\begin{abstract}
The detailed investigation of blast phenomena and their catastrophic effects on existing structures are the main objectives of the present paper. It is well known that blast phenomena may be characterized by significant complexity, often involving complicated wave propagation effects as well as distinguishable material behaviors. Considering the above and in an attempt to provide a simplified modelling approach for the simulation of blast effects, a novel procedure is presented herein based on well-established methodologies and common engineering practices. In the above framework, firstly, the "predominant" deformation shape of the structure is estimated based on elastic finite element simulations under blast loads and then the structural response of the system is evaluated as a result of common computational beam-element tools such as displacement-based pushover analysis. The proposed methodology provides an immediate first estimation of the structural behavior under blast loads, based on familiar engineering procedures. A two-span reinforced concrete bridge was thoroughly investigated and the results provide insightful information regarding the damage patterns and localization.
\end{abstract}

\section{Introduction}

Although blasts are considered phenomena of significant severity and potential socioeconomic impact, only recently did the authorities realize the necessity for the formulation of an integrated design and assessment protective framework. Modeling the effects of these phenomena on structures is very demanding, requiring highly sophisticated simulations including advanced constitutive material models. These procedures are resource- and time-consuming. On the other hand, there is a lack of simplifying procedures that could be implemented by practicing engineers through the utilization of common computational tools. Herein, a simplifying procedure is proposed based on common analytical and computational tools that would provide a preliminary but yet reliable estimation of the blast impact to the structural integrity of bridges.

Blasts are short duration dynamic events that generate dynamic pressure waves which propagate radially from the source in space, exciting dynamic response in the structures that are encountered in their path. The pressures acting on the affected surfaces are impulsive loads that impart significant amount of potential energy which sets damage-causing vibrations in the structure.

The various loads that act on a structure during its lifetime (natural or man-made) are characterized [1] by their range of frequency content and intensity (Figure 1(a)). Dynamic pressures exerted by blast explosions are considered among the most critical loads owing to their high intensity and frequency content, which falls within the range of values associated with fundamental eigenvalues of common buildings. Being a conveyer of potential energy, the blast wave evolves dilating in space (Figure 1(b)) as soon as it emerges. During this process it gets reflected on any surfaces it encounters when colliding with objects or structures or the ground (Figure 1(b)). In the case of surface explosions (where the source is at ground level), the reflection takes place almost simultaneously with the genesis of the wave. A schematic representation of the translation and dilation of the blast wave front caused by a surface explosion on a given structure are illustrated in 


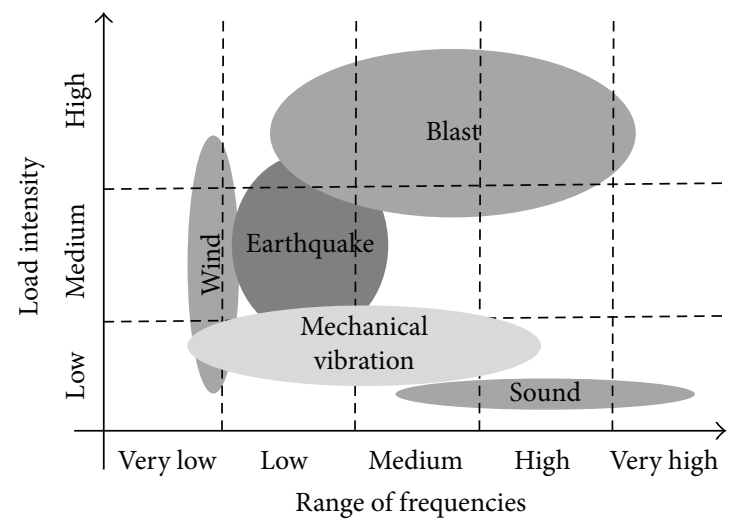

(a)

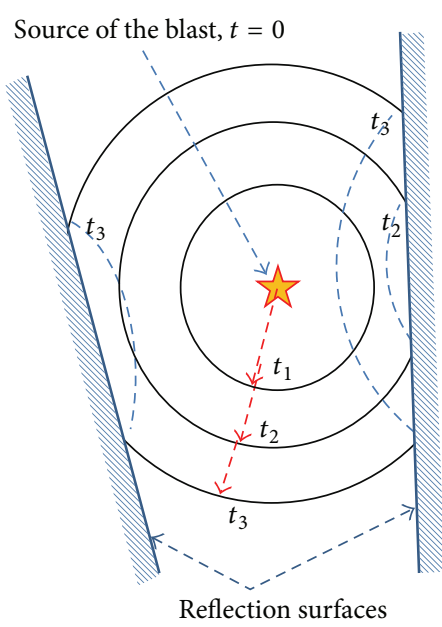

(b)

FIGURE 1: (a) Frequency range of different dynamic loads (according to [1]). (b) Collision and reflection of aerial blast waves $\left(t_{i}\right.$ are arrival times of the expanding wave front).

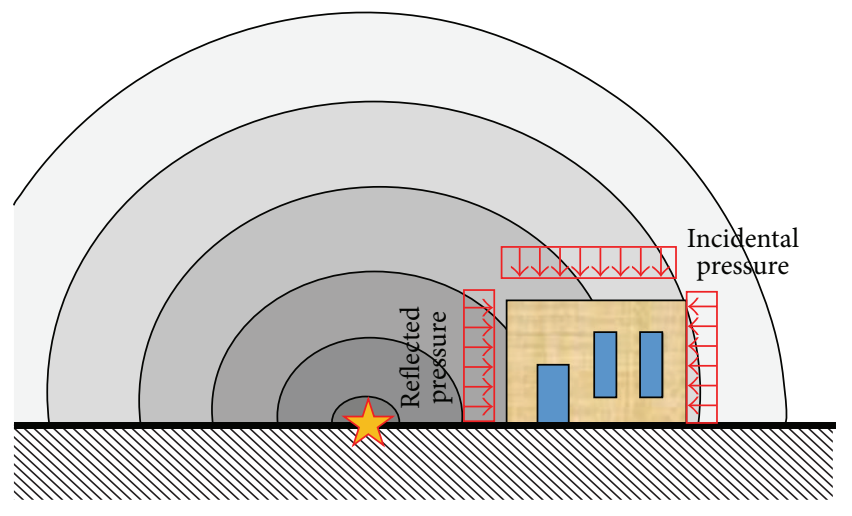

FIGURE 2: Schematic representation of the evolution of a surface blast wave in space; reflected and main pressure blast wave on an adjacent structure.

Figure 2. Note that reflected pressure waves affect surfaces perpendicular to the direction of the blast wave, whereas the incidental pressure wave affects the sides and back-face of the structure.

The effects of explosions are studied in this paper based on a simplifying procedure, with particular emphasis on one type of structure whose operation is vital in emergencies, namely, reinforced concrete highway overpasses. In the simplifying framework, the structure is approximated using concepts from generalized single-degree-of-freedom systems (Clough and Penzien [2]), where the dynamic response of the continuous structure is examined in a predominant shape of response. The proposed two-step methodology utilizes wellestablished structural engineering procedures as a preliminary design and assessment tool for the estimation of the impact of blast effects on structures. According to the above, the predominant deformation shape under blast loading is firstly evaluated based on ordinary elastic FE analyses and then a displacement-based pushover analysis is conducted to a simplified beam-element lumped plasticity model in order to evaluate the nonlinear behavior of each structural component for the induced deformation pattern of the previous step. With the above proposed procedure, the complicated blast problem is reduced to a simplified two-step procedure, which may be further simplified if certain characteristic deformation patterns for design purposes would be instantly provided in the future, based on the structural configuration and the blast initiation point.

The first section of the study deals with the mathematical definition of the excitation function exerted by the explosion and its numerical representation in the framework of established structural analysis software, considering both the temporal and spatial definition of the evolving pressure profiles acting on the exposed structural surfaces. The investigation uses a selected bridge case study subjected to a passing blast wave for illustration of concepts. A linear 3D finite element model of the bridge structure was combined with a consistent time history simulation of the explosion pulse, placing particular emphasis on the sequence of contact of the pressure wave with the structure, which depends on the physical distance and the location of the source relative to the structure. An essential ingredient for assessment of deformation demands and damage potential caused by the event is the deformed shape assumed by the structure through the time history of the event; this is extracted from the calculated response results. Next, using this pattern of deformations, the structure was analyzed based on the implementation of a simplified ordinary beam-element structural model so as to enable the use of the results of a nonlinear pushover analysis of the bridge under transient pressure profiles that simulate the explosive loads. This simplification alleviated partly the complexity of the problem associated with the time dependency of the constitutive properties of the materials, whereas it was possible to identify the tendency for damage localization throughout the structure. In this way, it is possible to take advantage of the nonlinear modelling technology that is 
stable and convergent when dealing with linear prismatic elements while avoiding convergence problems that would be owing to brittle failures in the continuous 3D finite element model.

\section{Effects of Blast Waves on Structures and Material Response}

2.1. Effects of Blast Waves on Structures. At any single point, the pressure wave has a time history of the type shown in Figure 3. The primary effect of a blast wave on a structure occurs during the positive phase, where pressure values are high; the negative phase has lesser consequences due to the attenuated pressure magnitudes and is usually neglected (Agrawal and Yi [1]).

An event is classified as blast if, compared with other catastrophic events such as wind and earthquake, the pressure momentum is several orders of magnitude greater than that of other phenomena (FEMA 426 [3]), whereas the pressure caused by the explosion attenuates quickly with increasing distance from the source affecting only a small part of the structure and causing great localized damage. Owing to the short duration of the blast, the mass participating in dynamic response is engaged usually at a later time, that is, until after the impulse has delivered its potential energy to its surroundings (positive phase). So, given that the loading has expired by the time the mass is mobilized in dynamic motion, it is extremely difficult to resonate the system while the load is acting. This definition underscores the difference between more conventional dynamic loads such as earthquake where partial resonance of eigenmodes is of primary significance. In the same context, due to the short duration of the loading exerted by the blast wave which typically ends within few milliseconds, it is possible to excite several additional modes (higher mode contribution) in the free vibration phase that follows, usually neglected in conventional seismic design. Of course this is a theoretical postulate; nonlinearity takes hold of the response as its intensity builds up, damping out quickly the participation of higher modes near failure.

The size and distribution of the applied pressures on the structure depend on the amount and type of released energy (which depend on the explosives used), the position of the source relative to the structure, and the magnitude and possible amplification of the resulting blast pressure owing to its interactions with objects encountered during dilation and propagation of the wave (Birhane [4]). The explosive wave exerts pressure on every exposed point of the structure encountered during its translation. The pressure depends on the arrival time $t_{a}$ of the wave front at the point of the structure considered. This time includes the time of transfer of the released energy from the explosive material to the environment (Martin [5]). The pressure rises from an initial value $p_{o}$ (the atmospheric or ambient pressure) to the peak normal pressure $p_{s o}$ (peak overpressure) instantaneously and it is subsequently reduced till its value attenuates to return to the initial pressure value. The durations of the positive and negative phases of the wave front are denoted by $t_{d}$ and $t_{n}$ (Figure 3): $t_{d}$ is the time of pressure attenuation from the peak value to the initial value, whereas $t_{n}$ is the subsequent time

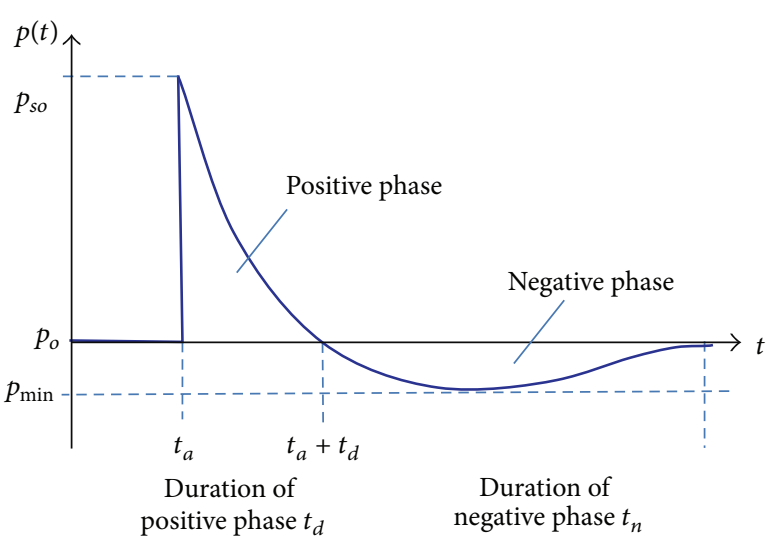

Figure 3: Diagram of pressure variation as a function of time.

interval up to the occurrence of the minimum negative value, $p_{\text {min }}$, in the negative phase. The pressure intensity versus time attenuation relationship is approximated by the following expression, known as the Friedlander equation (Baker [6]). The positive phase of the phenomenon concerns the main pressure wave of the blast wave (Figure 3):

$$
\begin{aligned}
p(t) & =p_{o}+p_{s o} \cdot\left(\frac{t-t_{a}}{t_{d}}\right) \cdot e^{-b\left(\left(t-t_{a}\right) / t_{d}\right)} ; \\
p_{s o} & =\frac{1}{Z} \cdot\left[\frac{1772}{Z^{2}}-\frac{114}{Z}+108\right](\mathrm{kPa}),
\end{aligned}
$$

where $Z=R /(W)^{1 / 3}$ is the scaled distance in $\mathrm{m} / \mathrm{kg}^{1 / 3}$ (obtained after pertinent normalizing; Ngo et al. [7]), $W$ is the amount of the explosive material in $[\mathrm{kg}]$, and $b$ is the coefficient of degradation which defines the slope of the attenuation curve (Martin [5]):

$$
b=5.2777 * Z^{-1.1975} \text {. }
$$

Variable $t_{d}$ (the positive phase duration) is defined by (Pandey et al. [8])

$$
\begin{aligned}
& \frac{t_{d}}{W^{1 / 3}} \\
& =\frac{980 \cdot\left[1+(Z / 0.54)^{10}\right]}{\left[1+(Z / 0.02)^{3}\right] \cdot\left[1+(Z / 0.74)^{6}\right] \cdot \sqrt{1+(Z / 6.9)^{2}}} .
\end{aligned}
$$

For hemispherical explosions, that is, explosions that occur on ground surface, it is recommended that the variable term in (1) be multiplied by a factor equal to 1.8 to account for the reflection of the blast wave which occurs during the explosion (Lam et al. [9]):

$$
p(t)=p_{o}+1.8 \cdot p_{s o} \cdot\left(\frac{t-t_{a}}{t_{d}}\right) \cdot e^{-b\left(\left(t-t_{a}\right) / t_{d}\right)}(\mathrm{kPa}) .
$$

2.2. Material Response under Strain Rate Loading. High strain rates affect the mechanical properties of the materials and thus the mechanisms of degradation of the various elements 
of the structure. The effect of high deformation rate on material strengths is quantified by the Dynamic Increase Factor (DIF), which is defined as the ratio of the dynamic to static strength (Javier and Allen [10]). Blast loading causes excessively high rates of deformation in the order of $10^{2} / \mathrm{s}-$ $10^{7} / \mathrm{s}$, whereas for usual loads the rate of deformation ranges between $10^{-7} / \mathrm{s}$ and $10^{0} / \mathrm{s}$ (for reference, note that the rates associated with Creep and Relaxation phenomena are in the range of $10^{-8} / \mathrm{s}$ to $10^{-5} / \mathrm{s}$; pseudostatic loads occur at strain rates from $10^{-8} / \mathrm{s}$ to $10^{-4} / \mathrm{s}$; earthquake loads occur at strain rates from $10^{-5} / \mathrm{s}$ to $10 / \mathrm{s}$. Explosions and collisions occur at strain rates from $10 / \mathrm{s}$ to $10^{7} / \mathrm{s}$, whereas even higher rates correspond to astrophysical phenomena, CEB-FIP, Bulletin $56[2])$.

For reinforced concrete structures subjected to blast loading, the strength of concrete and reinforcing materials may experience a significant increase due to the rate effects. The increase may exceed $50 \%$ for the reinforcing steel, whereas it may exceed $100 \%$ for concrete in compression and more than $600 \%$ for concrete in tension (Javier and Allen [10]). An implication of this disproportional strength increase of the two materials is an alteration in practice of the intended hierarchy of failure modes: thus, a design controlled by flexural yielding under relatively low rates of loading may become controlled by shear or by a combined shear-flexural mode of failure at higher rates (Ngo et al. [7]). Design charts are available to estimate the DIF for the two materials based on the strain rate (CEB-FIP, Bulletin 55 [2]); however, the phenomena may be also approximated by the following equations.

2.2.1. Rate Effects on Concrete Strength in Compression. Consider

$$
\begin{aligned}
& \mathrm{DIF}=\frac{f_{c, i m p, k}}{f_{c m}}=\left(\frac{\dot{\varepsilon}_{c}}{\dot{\varepsilon}_{c o}}\right)^{0.014}, \text { for } \dot{\varepsilon}_{c} \leq 30 \mathrm{~s}^{-1} \\
& \mathrm{DIF}=\frac{f_{c, i m p, k}}{f_{c m}}=0.012 *\left(\frac{\dot{\varepsilon}_{c}}{\dot{\varepsilon}_{c o}}\right)^{1 / 3},
\end{aligned}
$$

$$
\text { for } \dot{\varepsilon}_{c}>30 \mathrm{~s}^{-1} \text {, }
$$

where $f_{c, i m p, k}$ is the dynamic compression strength for loading rate $\dot{\varepsilon}_{c}, f_{c m}$ is the static compressive strength at loading rate $\dot{\varepsilon}_{c o}, \dot{\varepsilon}_{c}$ is the rate of deformation taking values in the range from $30 \times 10^{-6} \mathrm{~s}^{-1}$ to $3 \times 10^{2} \mathrm{~s}^{-1}$, and $\dot{\varepsilon}_{c o}=30 \times 10^{-6} \mathrm{~s}^{-1}$ is the rate of deformation under pseudostatic compression loading.

\subsubsection{Rate Effects on Concrete Strength in Tension. Consider}

$$
\begin{aligned}
& \mathrm{DIF}=\frac{f_{c t, i m p, k}}{f_{c t m}}=\left(\frac{\dot{\varepsilon}_{c t}}{\dot{\varepsilon}_{c o}}\right)^{0.018}, \text { for } \dot{\varepsilon}_{c t} \leq 10 \mathrm{~s}^{-1} \\
& \begin{aligned}
\mathrm{DIF}=\frac{f_{c t, i m p, k}}{f_{c t m}}=0.0062 *\left(\frac{\dot{\varepsilon}_{c t}}{\dot{\varepsilon}_{c t o}}\right)^{1 / 3}, & \\
& \text { for } \dot{\varepsilon}_{c t}>10 \mathrm{~s}^{-1} .
\end{aligned}
\end{aligned}
$$

Term $f_{c t, i m p, k}$ in (5a) and (5b) is the dynamic tensile strength for loading rate $\dot{\varepsilon}_{c t}, f_{c t m}$ is the static tensile strength for loading rate $\dot{\varepsilon}_{c t o}, \dot{\varepsilon}_{c t}$ is the rate of tensile deformation in the range from $1 \times 10^{-6} \mathrm{~s}^{-1}$ to $3 \times 10^{2} \mathrm{~s}^{-1}$, and $\dot{\varepsilon}_{\text {cto }}=1 \times 10^{-6} \mathrm{~s}^{-1}$ (the rate of deformation at pseudostatic tensile loading conditions). Apart from material strength, the high deformation rate affects all other mechanical properties. For example, by definition, the modulus of elasticity of concrete under high deformation rate may be estimated from the following equation:

$$
\frac{E_{c, i m p}}{E_{c i}}=\left(\frac{\dot{\varepsilon}_{c}}{\dot{\varepsilon}_{c o}}\right)^{0.026},
$$

where $\dot{\varepsilon}_{c}$ is the rate of deformation under dynamic loading, $E_{c, i m p}$ is the dynamic modulus of elasticity, and $E_{c i}$ is the modulus of elasticity of concrete. Combining the above equations (and considering the familiar Hognestad's parabola for the stress strain response in uniaxial compression), the strain at peak stress considering the modulus and strength dynamic enhancement are estimated from

$$
\frac{\varepsilon_{c 1, i m p}}{\varepsilon_{c 1}}=\left(\frac{\dot{\varepsilon}_{c}}{\dot{\varepsilon}_{c o}}\right)^{0.02},
$$

where $\varepsilon_{c 1, i m p}$ is the deformation at peak stress for a load rate $\dot{\varepsilon}_{c}$ and $\varepsilon_{c 1}$ is the deformation at peak stress under pseudostatic load.

2.2.3. Coefficient of Dynamic Amplification for Steel. Javier and John [11] studied the strength increase of reinforcement through experimental testing under high strain rates. Results followed a nonlinear relationship, where the rate of change of the dynamic amplification increased linearly with strain rate. It was concluded that the logarithm of DIF is at a linear relationship with the logarithm of the strain rate, $\dot{\varepsilon}$. The relationship that was used, both for the yield stress and for the ultimate strength, is (Javier and John [11])

$$
\mathrm{DIF}=\left(\frac{\dot{\varepsilon}}{10^{-4}}\right)^{a}
$$

Parameter $a$ used for the calculation of the yield and ultimate reinforcement stress is obtained from the following relationships:

$$
\begin{aligned}
& a=a_{f_{y}}, \quad \text { where } a_{f_{y}}=0.074-0.04 \cdot\left(\frac{f_{y}}{414}\right) \\
& a=a_{f_{u}}, \quad \text { where } a_{f_{u}}=0.019-0.009 \cdot\left(\frac{f_{y}}{414}\right) .
\end{aligned}
$$

$f_{y}$ is the yield stress of the reinforcement and $f_{u}$ is the ultimate strength of the reinforcement in MPa.

This model applies to reinforcing steel with yield stress ranging between 290 and $710 \mathrm{MPa}$ and for strain rates ranging between $10^{-4}$ and $225 \mathrm{~s}^{-1}$. The strength increase estimated according to (9a) and (9b) is considered only for passive reinforcement (i.e., it is not valid for prestressing streel. Dynamic amplification is not considered when dealing with the strength of prestressing cables). 


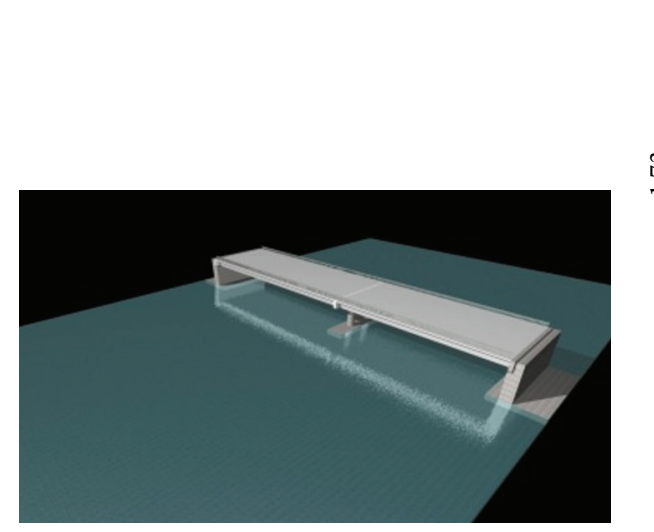

(a)

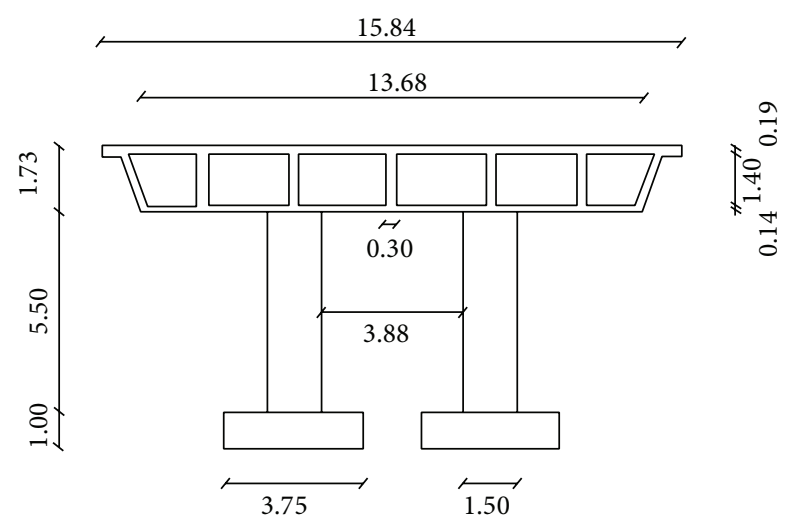

(b)

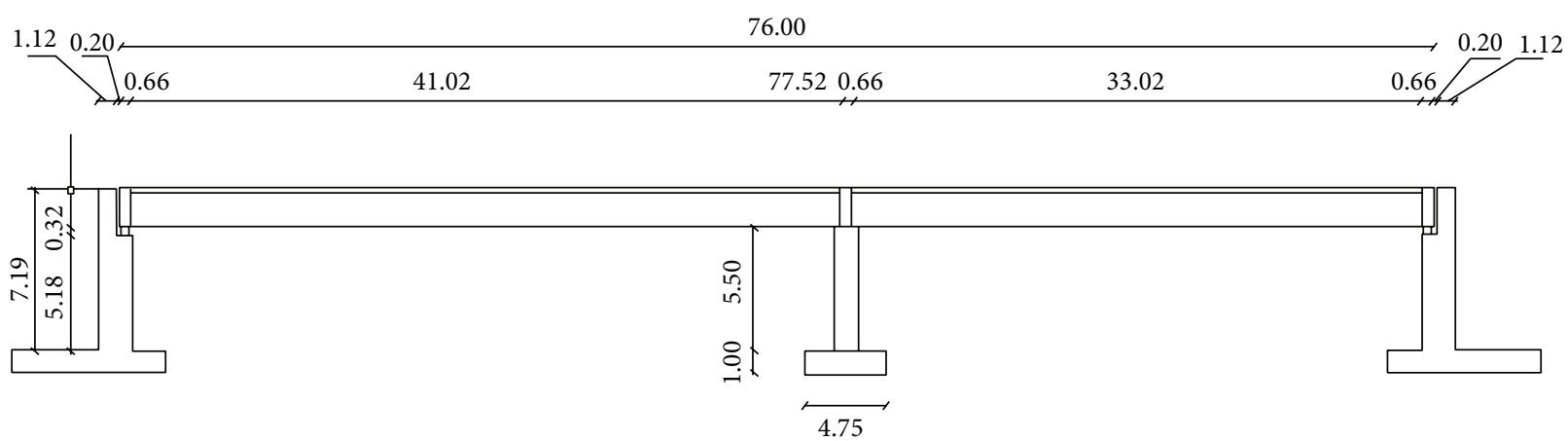

(c)

FIGURE 4: (a) Schematic representation of the bridge. (b) Geometric characteristics (transverse cross section). (c) Lengthwise cross section of the bridge.

\section{Investigating the Effects of Blast Waves on a Highway Overpass}

Blast effects on bridges are studied on a model bridge highway overpass, as illustrated in Figure 4(a). The bridge superstructure selected for study has the geometric properties of an actual bridge system (Kotsoglou and Pantazopoulou [12]) with monolithic connection at the central bent and the edge abutments (Figure 4(b)). The bridge has two unequal spans with a two-column central bent. The deck comprises a box section of multiple cells. Span lengths are $42.00 \mathrm{~m}$ and $34.00 \mathrm{~m}$ (Figure 4(c)). The box cross section is divided to cells through 5 beams of I cross section, having a web width of $0.3 \mathrm{~m}$, whereas the superstructure height is $1.73 \mathrm{~m}$.

The central bent columns have a circular cross section of $1.50 \mathrm{~m}$ diameter supported by separate pile caps resting on pile groups; each foundation block was a $3.75 \mathrm{~m} \times 4.75 \mathrm{~m} \times$ $1.00 \mathrm{~m}$ rectangular block. Clear height of the columns was $5.50 \mathrm{~m}$. The portal frame bent was centered at the midpoint of the deck width with no eccentricity, whereas the clear transverse distance between columns was $3.88 \mathrm{~m}$. Columns were monolithically connected with a bent cap beam (transverse beam) which in turn was connected monolithically with the adjacent deck superstructure. In the analysis of the bridge case study, the following parameter values were assumed: C30/37 concrete class (Eurocode 2, 2004) with a characteristic compressive strength $f_{c k}=30 \mathrm{MPa}$ and a nominal elastic modulus $E_{c}=32 \mathrm{GPa}$, whereas $S 500 B$ steel was taken for all loose reinforcement (characteristic steel yield strength $f_{y k}=$ $500 \mathrm{MPa}$ ). Self-weight and support reactions were estimated from the available architectural drawings of the bridge.

3.1. Variation of Simulated Blast Pressure on the Bridge. The problem considered in this study concerns the dynamic response of the bridge described above, owing to a surface explosion at near distance. A key issue to resolve first is the pressure profile occurring throughout the bridge structure and how the pressure wave propagates in space and time.

At the moment of the explosion the main pressure wave is transmitted uniformly in all directions. Over the duration of the transmission process the pressure varies with time: the peak pressure that occurs in each point in space depends on the distance from the source and the amount of the explosive material (Figure 5). It has therefore, for each explosion, the same value for all the points that are located at specific distance from source (spherical distance from the source as illustrated in Figure 2). In this representation $p_{s o 1}, p_{s o 2}, p_{s o 3}$, and $p_{s o 4}$ are the peak values of the blast wave at distances $R_{1}$, $R_{2}, R_{3}$, and $R_{4}$ from the source for the respective arrival times $t_{1}, t_{2}, t_{3}$, and $t_{4}$. Note that the values of the peak pressure are inversely proportional to the normalized distance, $Z$. For small values of normalized distance the pressures that 


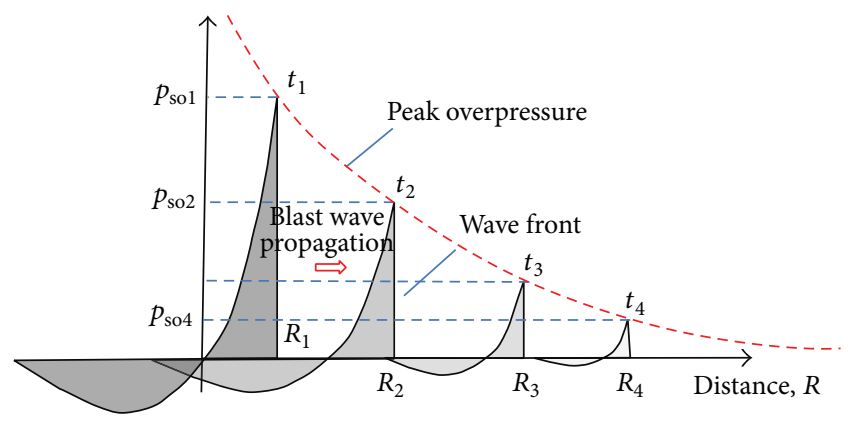

Figure 5: Attenuation of peak overpressure with distance from source: blast wave propagation.

develop are much higher relative to the peak pressures at points at a greater normalized distance.

Furthermore, the pressure of the blast wave does not attenuate at the same rate in all points in space since it depends on the coefficient of degradation $b$ which is a function of $Z$. It is easy to demonstrate that the value of $b$ increases, while the normalized distance decreases; that is, for small distances from the source and large amounts of explosive matter the coefficient is large, and the pressure decays quickly. This is the physical significance of $b$, whereas its calculation is related to the impulse (potential energy release) of the blast wave.

The effect of the pressure on the structure begins at the instant when the blast wave arrives at a specific point on its exposed surface, denoted henceforth as the "arrival time." Pressure value is maximum for any given spatial point in consideration at the arrival time. The pressure magnitude attenuates from that peak value according to (1).

The duration of the time period over which the pressure values exceed the atmospheric pressure also depends on $Z$. The so-called positive phase begins at the time of arrival and ends at the instant where the pressure becomes equal to the atmospheric value for the first time. The instantaneous equilibrium that takes place heralds the beginning of the second phase of the phenomena, referred to as "negative phase." The negative phase has a significantly longer duration as compared with the positive phase; however, its implications on the structure are significantly lower and often are neglected, while the values assumed by the negative pressure are comparatively much lower than those of the positive phase. The wind suction that follows the blast wave during its motion is related to the dynamic pressure, with the value always being equal to or greater than the atmospheric one (i.e., it does not assume negative values).

Regarding the bridge under consideration, the source of the explosion was assumed to be located on ground surface. Thermal radiation released upon the explosion and its effects on the material response were ignored in the present study. Surfaces that lie on the path of the wave front receive the reflected pressure, whereas the others receive the incidental pressure (Figure 2). To calculate the reflected pressure a coefficient equal to 1.8 was used on peak pressure in the Friedlander equation (see (6)). Material properties used in dynamic analysis of the FE model of the bridge were adjusted to account for the effects of dynamic amplification; note that for blasts of moderate and high intensity (i.e., for strain rates higher than the reference value of $3 \times 10^{2} \mathrm{~s}^{-1}$ ) the DIF may be estimated from (4a)-(9b). For the case study considered herein the assumed explosive material was $500 \mathrm{Kgs}$ TNT at a transverse distance of $3 \mathrm{~m}$ from the bridge's central bent (Figure 6(a)).

\section{Methodology in Modelling the Blast Wave}

In the present section, a two-step simplifying methodology for the evaluation of blast effects on structures is proposed and implemented. The main scope is to provide an approximate yet reliable tool for instant estimations of the above effects based on well-established engineering design and assessment strategies. According to the methodology, a corresponding predominant deformation shape is initially estimated based on a simplified elastic analysis to be used as a displacement loading pattern in a detailed lumped plasticity beam-element computational model of the bridge [13]. The latter is considered to be a displacement-based pushover analysis in that instead of a fixed pattern of loads of gradually increasing intensity, the structure is subjected to a fixed pattern of displacements of gradually increasing intensity. The target displacement intensity level is that attained in the elastic analysis when accounting explicitly for the pressure front evolution. The above simplification is advantageous, not only because many engineers worldwide are familiar to the "pushover analysis" concept, but also because it reduces a multiparametric problem into a simplified structural engineering problem. Furthermore, when compared with detailed FE inelastic simulation models, a wide range of computational shortcomings may be eliminated, such as convergence due to ill-conditioning of material stiffness after brittle cracking, time and resource consuming problems, and so forth. Herein, the corresponding deformation shape of the system, under blast loads, was evaluated based on an elastic, timedependent FE model of the case study bridge [14], but note that simplified, rational deformation shape approximations could be used instead.

Therefore, based on the deformation shape pattern obtained in the initial step, a displacement-based pushover analysis is conducted on a detailed beam-element computational simulation. Lumped plasticity or even brittle failure [13] properties (e.g., moment-curvature and shear failure criteria) were incorporated to each structural element in order to account for the induced damage.

4.1. Calculating the Load of the Blast Wave Front. Let time be defined with the reference starting point set at the instant of the explosion $\left(t_{i}=0\right)$; at that instant the wave begins to dilate in space evolving in all directions (Figures 1 and 6(a)). At time $t_{a}$ the blast front reaches the nearest point of the structure (the point at shortest travel distance from the source; Figures 6(b) and 6(c)). In the bridge case study and for the blast event under consideration, this point is at the base of the central bent on the exposed side. At time $t_{1}=t_{a}$ during which the blast wave has come in contact with the structure, the column base at the central pier support is the only point subjected to 


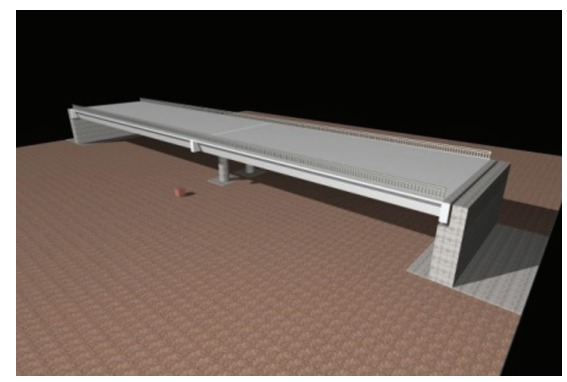

(a)

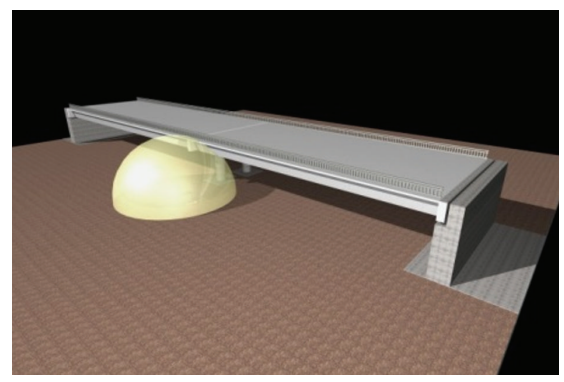

(d)

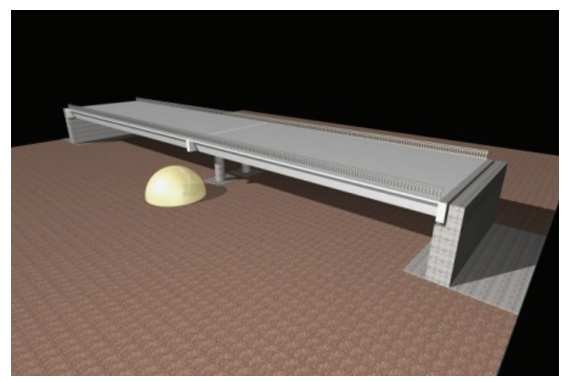

(b)

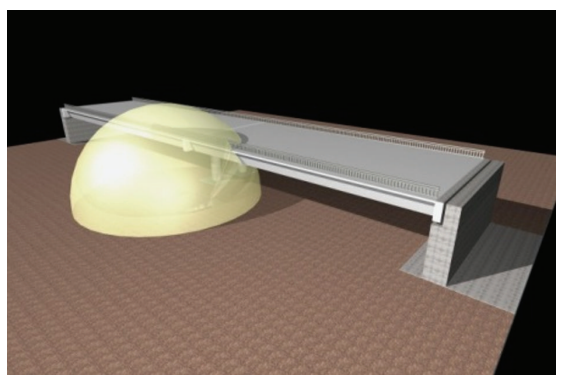

(e)

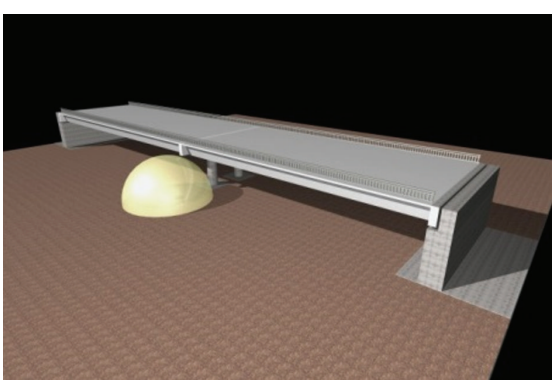

(c)

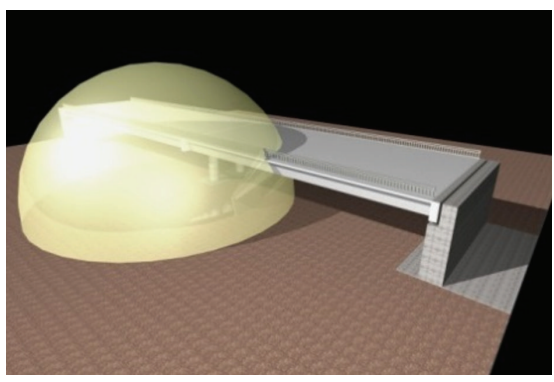

(f)

FIGURE 6: Schematic representation of contact of the blast wave with the bridge superstructure.

normal pressure. The intensity of this pressure applied at time $t_{1}$ on this point is equal to $p_{s o 1}$ (Figure 5), whereas the rest of the structure remains unloaded.

During time $t_{2}>t_{1}$, the wave has dilated and comes in contact with a new surface (loading surface) which is defined by the common points that lie on the cross section of the sphere that represents the wave front and the structure (Figure 6(d)) and are also at a distance greater than that of the initial point of contact. This distance depends on the speed of evolution of the blast front. At this instant of time this specific surface is loaded by pressure $p_{s o 2}$ (Figures 6(c)-6(f)). Simultaneously the initial point is loaded by pressure $p_{1}<$ $p_{s o 1}$, where $p_{1}$ is calculated according to the curve of Figure 3, for $t=t_{2}$. Such surfaces, as they have not been met by the blast front at the time in consideration, remain unloaded.

The same thing holds at time instances, $t_{3}, t_{4}, \ldots, t_{n}$, when the peak pressures occur, $p_{s o 3}, p_{s o 4}, \ldots, p_{\text {son }}$, for $n$ surfaces as they come progressively in contact with the wave front, whereas surfaces through which the wave has already passed are loaded by attenuated pressure magnitudes. These are estimated by the model of pressure variation as shown in Figure 3 after introducing properly the parameter values. Thus each new surface of the structure which is intersected by the spherical wave front is loaded by the peak pressure at the instant of contact, whereas afterwards it continues to be loaded by the pressure estimated from the attenuation relationships until it becomes eliminated.

The set of contact points which are loaded simultaneously is evaluated automatically by calculating their normalized distance from the source based on the assumption that the blast wave evolves at constant speed radially (the geometric distance and the amount of the explosive matter are combined to calculate the normalized distance; this is then introduced in the corresponding relationships for calculation of pressure at every point in a given time). As illustrated in Figures 6(c)-6(f), there are surfaces in different elements of the structure that are loaded simultaneously.

The speed of the wave front is calculated from the following equation (Ngo et al. [7]):

$$
v_{s}=a_{o} \cdot\left(\frac{6 p_{s o}+7 p_{o}}{7 p_{s o}}\right)^{1 / 2}
$$

where $p_{s o}$ is the peak positive pressure, $p_{o}$ is the ambient atmospheric pressure before the explosion, and $a_{o}$ is the speed of sound at the ambient conditions.

The magnitude of the pressure felt by each surface depends on its position relative to the direction of evolution of the wave front. Surfaces located at the forefront of the wave transmission receive the reflected pressure, whereas all other surfaces receive the incidental pressure (Figure 2).

Calculating in this manner the pressure that acts on the various points of the structure in time, the problem is reduced to a classical problem of structural dynamics which may be solved numerically using established procedures (e.g., with finite elements); the boundary of each finite element lying on the perimeter of the structure is loaded by a time-varying pressure function depending on its distance from the source. The volume of data that must be calculated is proportional to the size and complexity of the structure, and thus significant computing capacity is required for a usual structure. To deal with this problem, in the present investigation, a collection of geometric loci representing the intersection of the advancing hemispherical wave front with the structure were determined at no loss of accuracy. Based on the consecutive, 

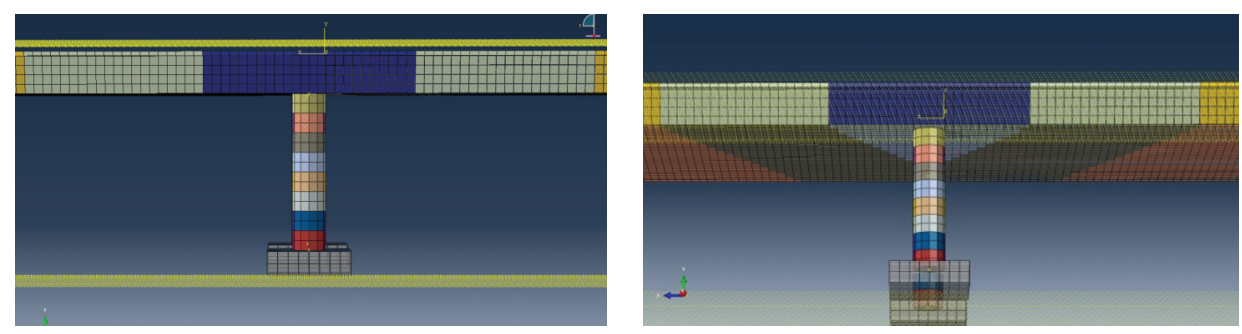

FIGURE 7: Separation of the loading surfaces of column and bridge deck to model the spherical propagation of the blast wave.

time-dependent contact patterns with the bridge depicted in Figure 7, discrete loading surfaces were defined on each critical structural component (i.e., columns and deck surfaces) in order to simulate the wave propagation with time. From the diagram of pressure variation with time depicted in Figure 3 and considering the wave propagation pattern of Figure 6, each loading surface is characterized by a discrete arrival time $t_{a}$ which depends on the distance from the source and the speed of the wave as estimated by (10). Figure 7 illustrates the loading surfaces defined on the bridge, whereas pressure was taken as constant within each step. The pressure value was set equal to the mean, that is, the value associated with the average distance of the points on the surface of the bridge to the source. For the column, surfaces had a smaller area as initially, after arrival, pressure shows a faster rate of attenuation, whereas for the deck these surfaces were substantially larger as, with increasing time, the rate of pressure reduction drops as illustrated in Figure 3. Based on the above, deck and column surfaces were defined symmetrically around the initial contact point and were discretized into five and eight segments, respectively (Figures 7 and 8(a)). Note that reflected pressure waves affect the surfaces (Figure 8(b)) at the forefront (i.e., front-face of the columns, bottom, and front-face of the bridge deck), whereas the incidental pressure wave affects the sides and back-face of the structure (backface of the columns, upper surface of the superstructure).

4.2. Proposed Simplifying Methodology. In the present section, a simplifying methodology is proposed for a fast evaluation of blast effects based on the utilization of common analytical tools. In general, such complicated phenomena as blast effects would demand modelling and analytical approaches with significant computational cost. The dynamic nature of the problem, in conjunction with the advanced material constitutive properties, would drive to extremely complicated FE models with very large number of nodes, significant computational cost, and questionable results (e.g., convergence problems during inelastic finite element analysis). Based on the above, a simplifying, easy to use methodology was developed herein in order to reduce the computational cost and at the same time provide reliable solutions based on widely used software packages. The core of the proposed methodology is to define the predominant deformation shape of the structure under the blast wave and then to proceed with ordinary pushover analysis based on simplified, ordinary structural beam-element models with lumped properties. The entire method may be summarized in the following steps:

(a) Based on the theoretical background presented herein (Section 2), evaluate all acting pressures on the existing structural elements and their distribution along the bridge deck and substructure.

(b) Evaluate the structural behavior of all critical components (columns and beams) through the implementation of established analytical methodologies (i.e., moment-curvature section diagrams, shear and axial tension/compression ultimate strength values). The above-mentioned values will be used as an input to the lumped plasticity beam-element model and at the same time will form the failure/yielding criteria for the selection of a valid deformation pattern on the FE model of the next step. Strain rate phenomena should be also considered herein for concrete and steel, based on the provisions of Section 2.2.

(c) Generate a finite element model (simplified or detailed) and, through the implementation of elastic analytical tools, find the predominant deformation shape of the structure due to blast wave effects at peak dynamic displacement response. The detailed $3 \mathrm{D}$ finite element modelling approach presented in Section 4.1 is considered to be the most reliable solution, while other less time consuming strategies would be also acceptable (e.g., the use of 2D elements in widely used commercial software). Considering that the deformation shape is intended for use during a pushover analysis, the selection should correspond to the failure/yielding state of the structure based on appropriate yielding/fracture criteria defined for each critical structural component in the second step.

(d) Using the evaluated predominant deformation shape of the previous step, proceed with the conventional displacement-based pushover analysis in a simplified beam-element structural model with lumped properties. Detailed section properties and interactions are necessary to be incorporated in the model, based on ordinary structural modelling strategies (Step (b)). In any case, the resulting total force should be equal to or less than the maximum estimated applied force extracted from the FE model. 


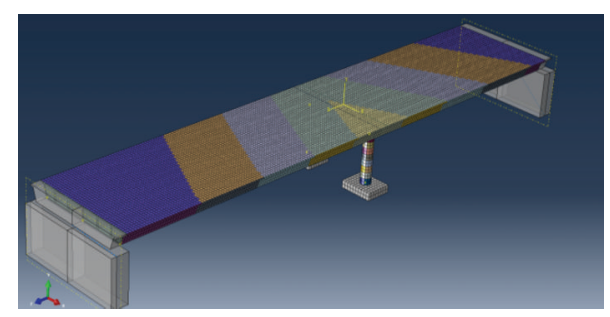

(a)

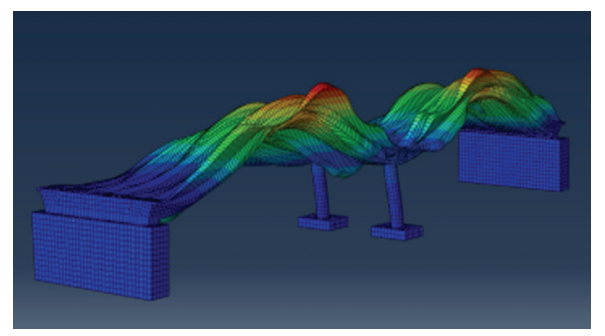

(c)

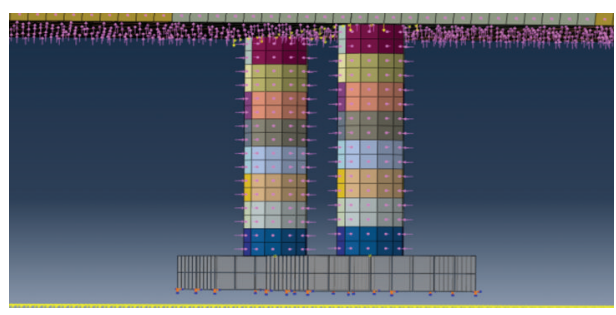

(b)

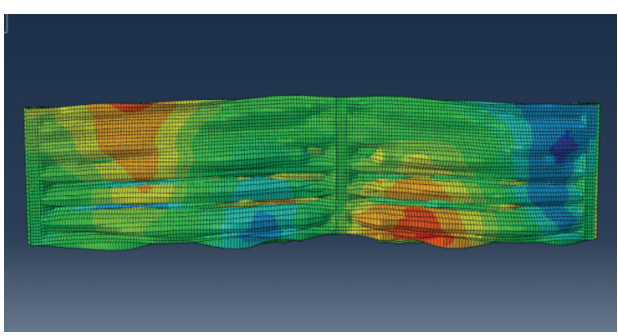

(d)

FIGURE 8: FE elastic analysis for a case with monolithic connections in the abutments and central pier. (a and b) Loading surfaces on the structure to model the wave transition. ( $c$ and d) Snapshots of the deformed state of the structure at the deck and around the central bent at peak displacement response.

4.3. Predominant Deformation Shape. A key issue for the implementation of the proposed methodology is the estimation of a representative, predominant deformation shape of the structure, under the induced blast loads. For this reason the "predominant" deformation shape is defined herein as the elastic deformation state of the structural system just before brittle failure or yielding of the selected, important for the stability, structural components. Considering the severity of the induced loads and the corresponding instant brittle failures in critical structural components, the above-mentioned deformation shape approximation usually provides representative patterns of force distribution along the structure. Note that local yielding of structural elements under blast loads is rare, while brittle failure modes are usually the dominant pattern of damage. According to the preceding, based on the anticipated failure characteristics of each critical structural component of the bridge (e.g., shear failure, axial force, or moment capacity), the elastic deformation shape is extracted from the implemented FE computational study. For the structural system under examination (post-tensioned concrete bridge), the critical components that control the entire response are the post-tensioned beams as well as the central bent columns and the dominant deformation shape of the system is depicted in Figures $8(\mathrm{c})$ and $8(\mathrm{~d})$.

4.4. Application to the Model of the Case Study and Results. As it has been already stated in Section 4.2, in order to minimize the computational cost, an elastic analysis (Figure 8(c)) was first conducted in order to determine the "predominant" deformation shape assumed by the structure which was then scaled to yielding in the plastic hinge regions of the central pier columns. The results of the analysis are illustrated in Figure 8 for the peak estimated response. The structure is initially at rest as shown in Figures 8(a) and 8(b), where the loading surfaces to which the structure has been subdivided are illustrated. Based on the results of the elastic analysis it was found that the most critical pattern of deformation assumed by the structure resembles the shape of the fundamental mode for shaking in the transverse direction (Figures 8(c) and $8(d))$. It was found that this pattern occurs while the explosive wave moving from the base pushes the entire structure upwards. In order to proceed with the implementation of the proposed methodology and to extract the response into the nonlinear range without excessive calculation complexity, the simplified nonlinear frame model was assembled for the analysis of the structure. Actually, using the proposed modelling approach, it is possible to reduce the size of the problem, so as to enable the assessment of blast effects on the bridge while using simplified constitutive laws and material properties. Based on the above, the structure was subjected to the evaluated predominant deformation pattern shown in Figure 8(c) after this had been normalized to the peak value so as to represent a shape function (considering the shape shown in Figure $8(\mathrm{c})$, it may be said here that an analysis resembling in principle the standard "pushover" was attempted, with the approach being motivated from earthquake engineering practices and intended to be used to blast events). Based on the above defined deformation pattern, the intensity of deformation was gradually increased using a step by step nonlinear static procedure. This enabled inelastic state determination at different intensities of displacement of the deck when deforming according to the pattern shown in Figure 8(c). In any case, the resulting total force should be equal to or less than the maximum estimated applied force extracted from the FE model.

Considering that the problem under investigation was reduced into a model comprising typical frame elements, appropriate inelastic hinge properties were evaluated for each 
structural component of the model. Based on well-known structural engineering evaluations, a wide range of possible constitutive behaviors (brittle or ductile) were modelled analytically for each existing critical component (shear and axial compression/tension ultimate forces, moment-axial load interaction diagrams, moment-rotation envelopes for lumped plastic hinges, and plastic hinge lengths and momentcurvature relationships for member sections where a mode detailed idealization was needed). Objective of this approach was to quantify the extent of damage in terms of values of established stress and strain resultants in the critical elements of the bridge (Figure 9).

From the pushover analysis results it was found that plastic hinges form quickly in the structure, the most immediate being those at the top and the base of the two pier columns. Upon further examination of the estimated values it was concluded that columns would fail by a combination of shear and axial tension (as a result of the upwards pressure applied in the underside of the deck) after flexural yielding in the ends of the members. Considering the brittleness of the estimated mode of failure, the anticipated damage in the column could be extensive depending on the intensity of the blast wave. Beyond that point, a sequential formation of plastic hinges at various points in the structure is observed, owing to the vertical component of the blast wave throughout the span. The deck beams near the abutments and the central pier attain shear failure over an extensive portion of the span; no flexural failure is observed at mid-span suggesting that shear failure precedes flexural modes leading directly to nonproportional damage and collapse.

For the case under consideration, it is evident that, in contrast with ordinary seismic events, blast actions induce excessive loads which are distributed based on significantly different patterns along the superstructure and the substructure elements. Early axial tension and shear brittle failures dominate the response of the entire system, while flexural yielding phenomena, which are commonly considered to be critical in earthquake design practices, seem to be of minor importance.

Based on the above, it is evident that common earthquake design and detailing practices do not cover at all blast actions, as the developed mechanisms are significantly different from those considered in conventional load combinations including earthquake. Therefore, it should be underscored that existing infrastructure elements which are designed based on ordinary structural/earthquake design practices and provisions are vulnerable to significant blast loads and that additional measures need to be taken (active or passive protection) in order to secure the structural integrity of those bridge systems that would be considered critical for continuous functionality in an emergency situation.

\section{Conclusions}

A simplified modelling procedure for the fast assessment of the effects of blast loads on bridges is proposed. The methodology is a versatile tool for first-order estimation of the effects of blast explosions on structures, which would otherwise require an extensive and complicated nonlinear time history analysis. First the evolving pressure wave front was
Results from inelastic analysis of bridge case study

Early failure of the column joints as a result of axial tension and shear

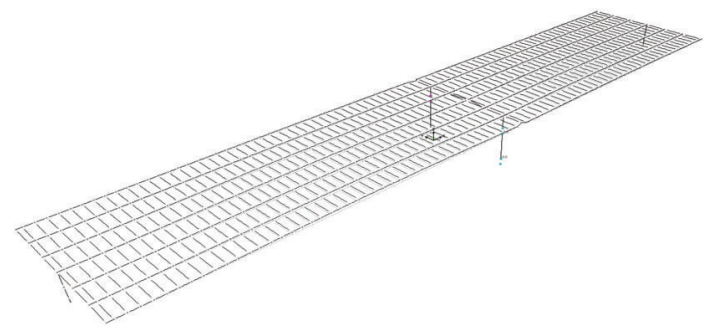

(1)

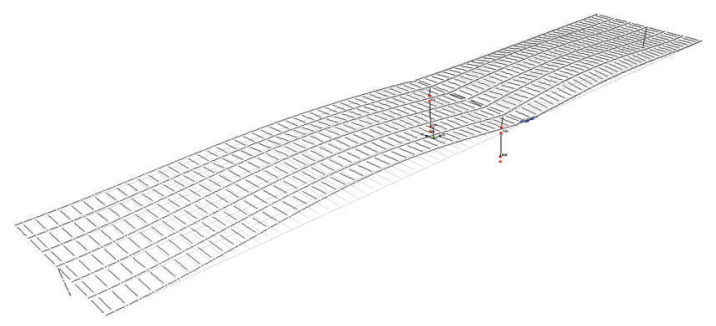

(2)

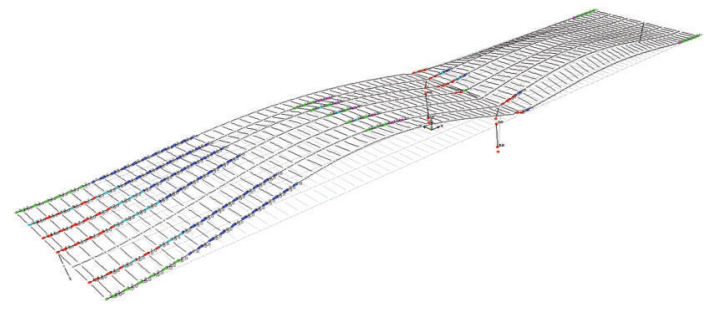

(3)

FIGURE 9: Analysis results for a case with fixed supports: monolithic connections in the abutments and central pier (obtained using nonlinear static analysis).

described mathematically in space and time so as to define its loci with the exposed structural surfaces. This enabled definition of the pressure forcing functions on the structure. This was applied using linear dynamic analysis on a detailed elastic finite element model of the structure in order to identify the predominant displacement profile experienced by the structure. Deformations were normalized to the peak response so as to develop a shape function for the bridge structure at the extreme displaced response. The identified displacement pattern was then applied on a nonlinear frame model of the structure. Through this modelling option, nonlinear static analysis was possible at a much reduced computational cost as compared to 3D solid nonlinear finite element modelling which is still, today, prohibitively time-consuming and computationally inaccessible when used to conduct time history investigation of the complete structure; a reason for this is lack of pertinent nonlinear cyclic brick FE models appropriate for modelling 3D solid reinforced concrete structures of realistic complexity, whereas brittle nonlinearity causes insurmountable convergence problems in $3 \mathrm{D}$ solid FE models. The frame model was subjected to the identified displacement pattern, with the intensity being increased gradually to 
the displacement levels identified by the elastic analysis. Note that the concepts used are extended from earthquake engineering where performance is established from a displacement-based pushover analysis up to the anticipated level of displacement demands. Nonproportional damage is the characteristic consequence of surface, near field explosions such as the example considered in the present study for a two-span typical highway overcrossing. It was found that axial tension as well as shear failure in the columns and deck owing to the excessive displacements caused by the blast having the intensity examined would lead to catastrophic collapse of the bridge. Clearly, blast actions induce severe loads on the structure which are distributed based on different patterns along the superstructure and the substructure elements. Considering that conventional structural and earthquake design fails to provide a solid framework against blast actions, additional measures are necessary to be implemented (e.g., active or passive protection of the system). Based on the simplicity and the reduced computational cost, the proposed method could form the basis for future investigations of blast phenomena as it is intended for a fast assessment procedure of structures subjected to accidental explosions.

\section{Competing Interests}

The authors declare that they have no competing interests.

\section{References}

[1] A. K. Agrawal and Z. Yi, Blast Load Effects on Highway Bridges, University Transportation Research Center, Department of Civil Engineering, The City College of New York, New York, NY, USA, 2008.

[2] R. W. Clough and J. Penzien, Dynamics of Structures, McGraw Hill, New York, NY, USA, 2nd edition, 1993.

[3] FEMA 426, Risk Management Series, Reference Manual to Mitigate Potential Terrorist Attacks Against Buildings, U.S. Department of Homeland Security, Southwest, Wash, USA, 2003.

[4] T. H. Birhane, Blast Analysis of Railway Masonry Bridges, University of Minho, Braga, Portugal, 2009.

[5] L. Martin, "Simulation of the effects of an air blast wave," JRC European Commission Technical Notes, 2007.

[6] W. E. Baker, Explosions in Air, University of Texas Press, Austin, Tex, USA, 1973.

[7] T. Ngo, P. Mendis, A. Gupta, and J. Ramsay, "Blast loading and blast effects on structures-an overview," Electronic Journal of Structural Engineering, vol. 7, pp. 76-91, 2007.

[8] A. K. Pandey, R. Kumar, D. K. Paul, and D. N. Trikha, "Nonlinear response of reinforced concrete containment structure under blast loading," Nuclear Engineering and Design, vol. 236, no. 9, pp. 993-1002, 2006.

[9] N. Lam, P. Mendis, and T. Ngo, "Response Spectrum Solutions for Blast Loading," Electronic Journal of Structural Engineering, vol. 4, 2004.

[10] M. L. Javier and R. C. Allen, "Review of strain rate effects for concrete in tension," ACI Structural Journal, vol. 95, no. 6, 1998.

[11] M. L. Javier and C. E. John, "Dynamic increase factors for steel reinforcing bars," in Proceedings of the 28th DDESB Seminar, Orlando, Fla, USA, August 1998.
[12] A. Kotsoglou and S. Pantazopoulou, "Response simulation and seismic assessment of highway overcrossings," Earthquake Engineering and Structural Dynamics, vol. 39, no. 9, pp. 991-1013, 2010.

[13] N. Priestley, F. Seible, and G. M. Calvi, Seismic Design and Retrofit of Bridges, John Wiley \& Sons, 1996.

[14] H. Hibbitt, B. Karlsson, and P. Sorensen, Abaqus Analysis User's Manual. Version 6.9, Dassault Systemes Simulia Corporation, Providence, RI, USA, 2010. 


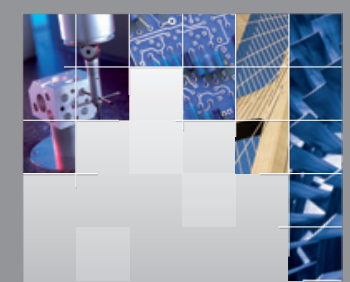

\section{Enfincering}
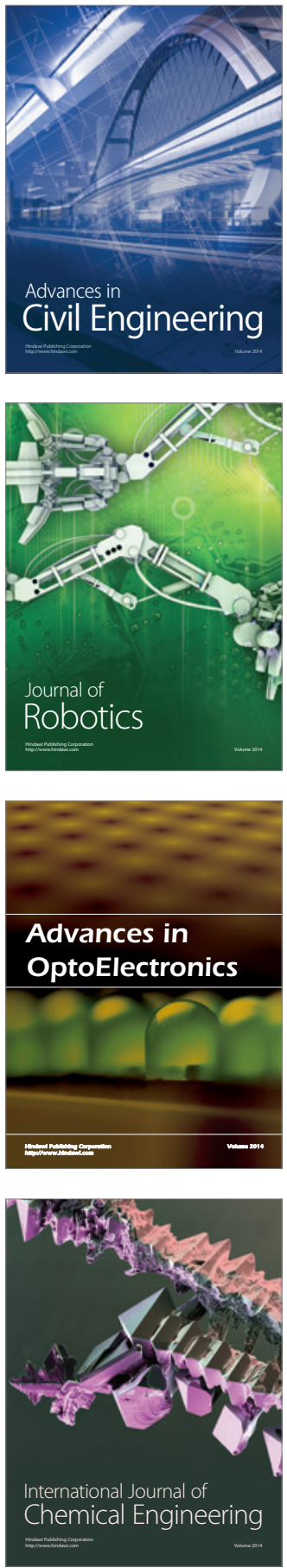

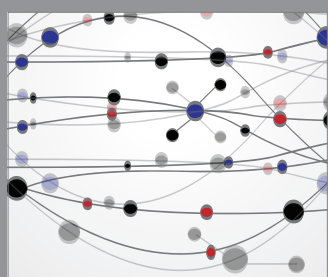

The Scientific World Journal

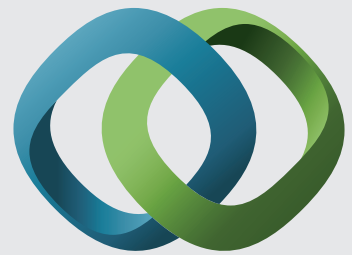

\section{Hindawi}

Submit your manuscripts at

http://www.hindawi.com
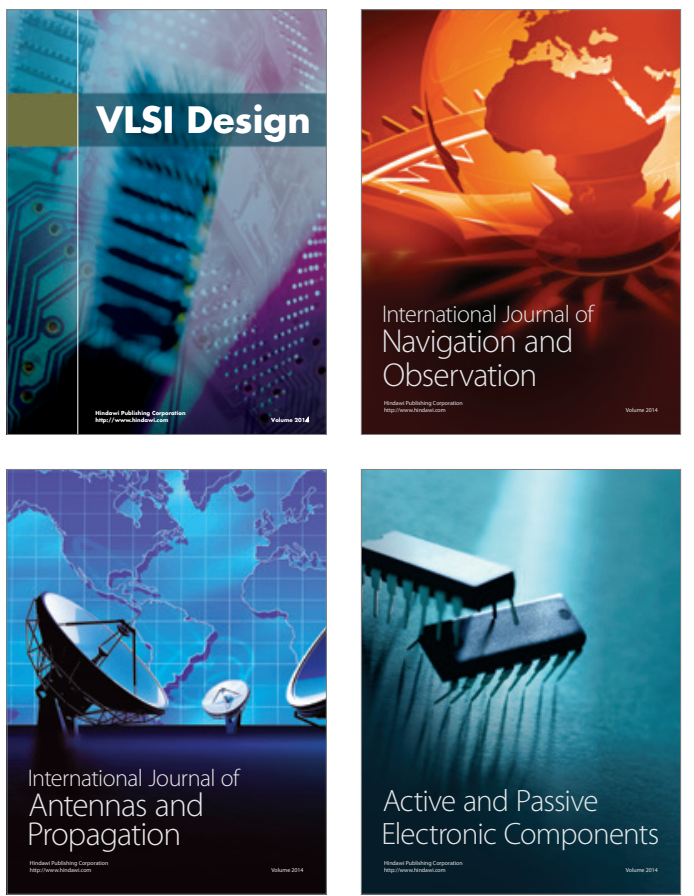
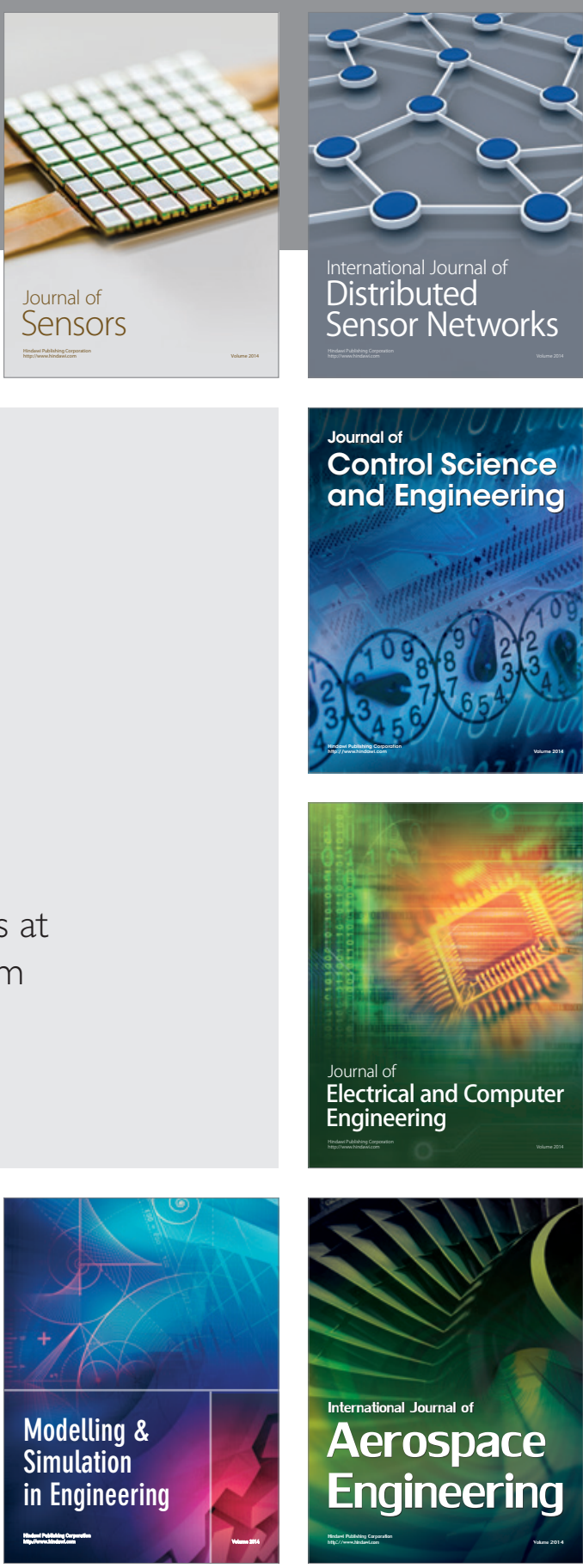

International Journal of

Distributed

Sensor Networks

Journal of

Control Science

and Engineering
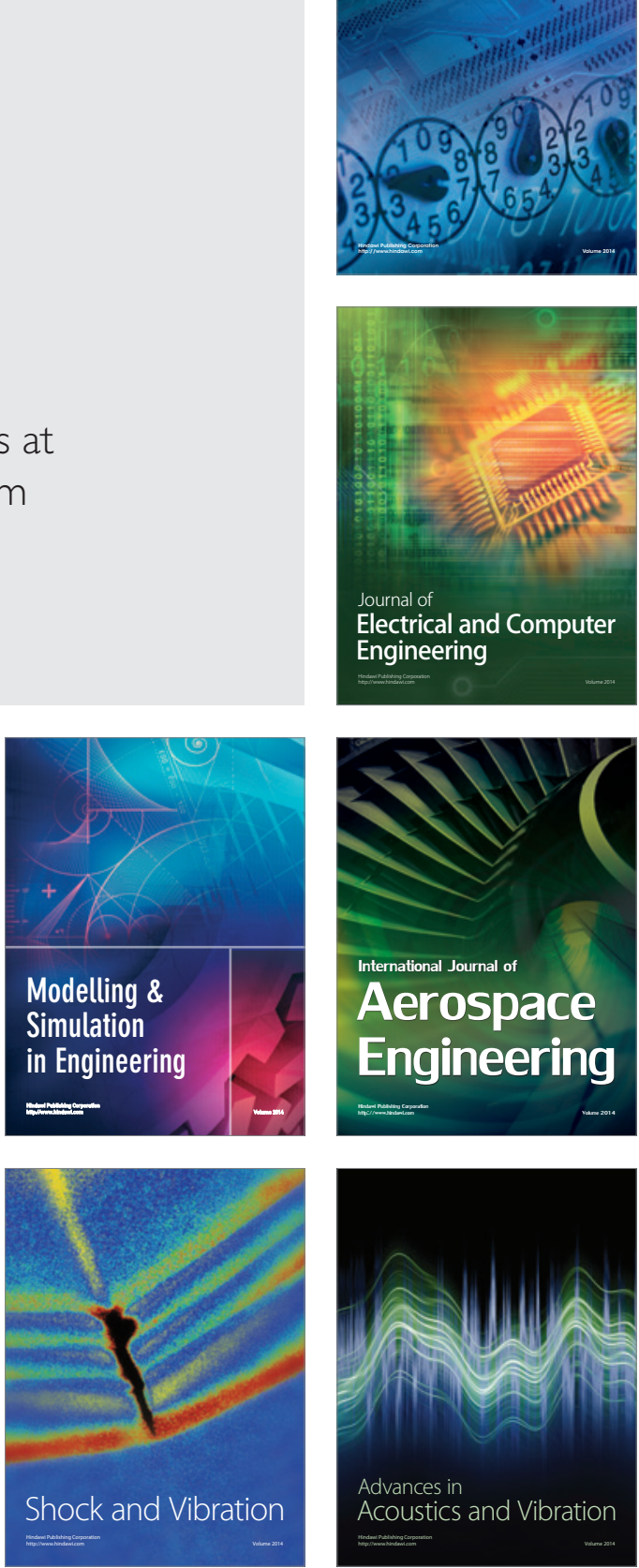